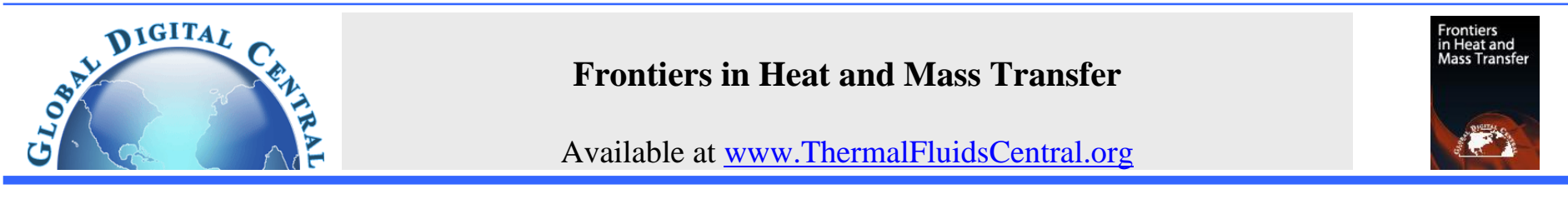

\title{
MELTING AND RADIATION EFFECTS ON MIXED CONVECTION BOUNDARY LAYER VISCOUS FLOW OVER A VERTICAL PLATE IN PRESENCE OF HOMOGENEOUS HIGHER ORDER CHEMICAL REACTION
}

\author{
D. R. V. S. R. K. Sastry \\ Department of Mathematics, SASTRA Deemed University, Thirumalaisamudram, Thanjavur, TamilNadu-613401, India
}

\begin{abstract}
The present paper investigates the combined effects of melting phenomenon and viscous dissipation over a steady incompressible mixed convection boundary layer fluid flow along a vertical plate. Radiation and double dispersion are also taken into consideration. Further effect of homogeneous chemical reaction of order ' $n$ ' is studied over the non-Darcy porous plate. Continuum equations that characterize fluid flow are transformed to a set of non linear ordinary differential equations through a suitable similarity transformation. These equations are then solved by MATLAB 'bvp4c' iterative programming method. As a matter of accuracy and validation, available results are compared with the present study as a special case. Flow characteristics of the problem are illustrated graphically.
\end{abstract}

Keywords: Chemical Reaction, Heat and Mass Transfer, Porous Medium, Melting Effect, Mixed Convection, Viscous Dissipation

\section{INTRODUCTION}

Heat and mass transfer over viscous geometries embedded in porous media has a considerable interest of many engineering and geophysical applications such as geothermal reservoirs, process of drying porous solids, Magnetohydrodynamics(MHD) power generators and underground energy transport. Considerable literature can be found over combined heat and mass transfer in free convection boundary layer flow over heated surfaces along different geometries. Few researchers (e.g., Gebhart et al., 1989; Kandasamy et al., 2006; Chaudhary et al., 2006), investigated the impact of radiation on heat transfer in MHD mixed convection flow with viscous dissipation and ohmic heating effects. A problem of analytical conjugate phenomena of heat and mass transfer was examined by Noor et al. (2012). A discussion on the influence of free convective effects on Stokes problem when the fluid is driven by applied magnetic field was focused by Soundalgekar et al. (1979). Effect of align magnetic field in a forced convection boundary layer flow was examined by Seddeek (2002). A study was made over mixed convection flow through permeable vertical surface in presence of radiation by Aydin and Kaya (2008).

The unique phenomena used in magma solidification, preparation of semi conductor materials and the systems where solid-liquid change materials is melting phenomena. Firstly, in absence of porous medium, Roberts (1958) examined the steady state effects when ice was placed in a hot stream of air. Later Epstein and Cho (1976) observed heat transport in a submerged bodies undergoing melting. Few authors explored the as- pects of the flow with melting phenomena (e.g., Gorla et al., 1999; Cheng and Lin, 2007). These studies revealed that melting process reduces the heat transfer through the solid-liquid interface. Effect of melting on mixed convective flow over a permeable vertical surface was feigned by Ahmad and Pop (2014). They found dual solution for some specific values of mixed convection parameter. The results also indicate that the melting phenomena reduces the heat transfer rate and expedites the boundary layer separation. Further Sobha et al. (2010) identified the nature of velocity across melting parameter in both aiding and opposing flows. Also in this study, it is observed that Nusselt number decreases with melting parameter and increases with increase in thermal dispersion. Prasad and Hemalatha (2010) examined the combined effects of radiation and melting over a vertical wall. Radiation effect on heat transfer in a flow over a vertical surface with uniform surface temperature was noticed by Hossain and Takhar (1996). Thermal dispersion and radiation effects on non-Darcy free convection flow through vertical plate were explained by Abbas et al. (2008). They observed that increasing radiation parameter enhances the momentum boundary layer thickness. Further Nusselt number increases with radiation parameter. Melting effect on a convective heat transfer between a melting body and surrounding fluid was discovered in Tien and Yen (1965). They further noticed that melting retards the rate of heat transfer.

It is evident from the literature that no work has been carried out on combined effects of viscous dissipation and melting phenomena in a 
mixed convection boundary layer chemically reacted fluid flow in presence of radiation over a permeable surface. So, in this paper, these effects on heat and mass transfer and as well as fluid velocity, temperature and concentration with in the boundary layer are investigated. The results are compared with Hemalatha et al. (2015) as a special case and found in good agreement.

\section{MATHEMATICAL ANALYSIS}

A problem of mixed convection steady state boundary layer flow through a vertical surface with melting phenomena is considered. Assume that the surface forms an interface between solid and liquid phases at the time of melting inside porous matrix. Plate is kept at constant temperature $T_{p}$. Further the temperatures $T_{s}$ and $T_{\infty}$ of solid phase and liquid phase far from the interface and plate respectively are assumed to be constant. Taking viscous dissipation along with radiation, the boundary layer equations are framed as

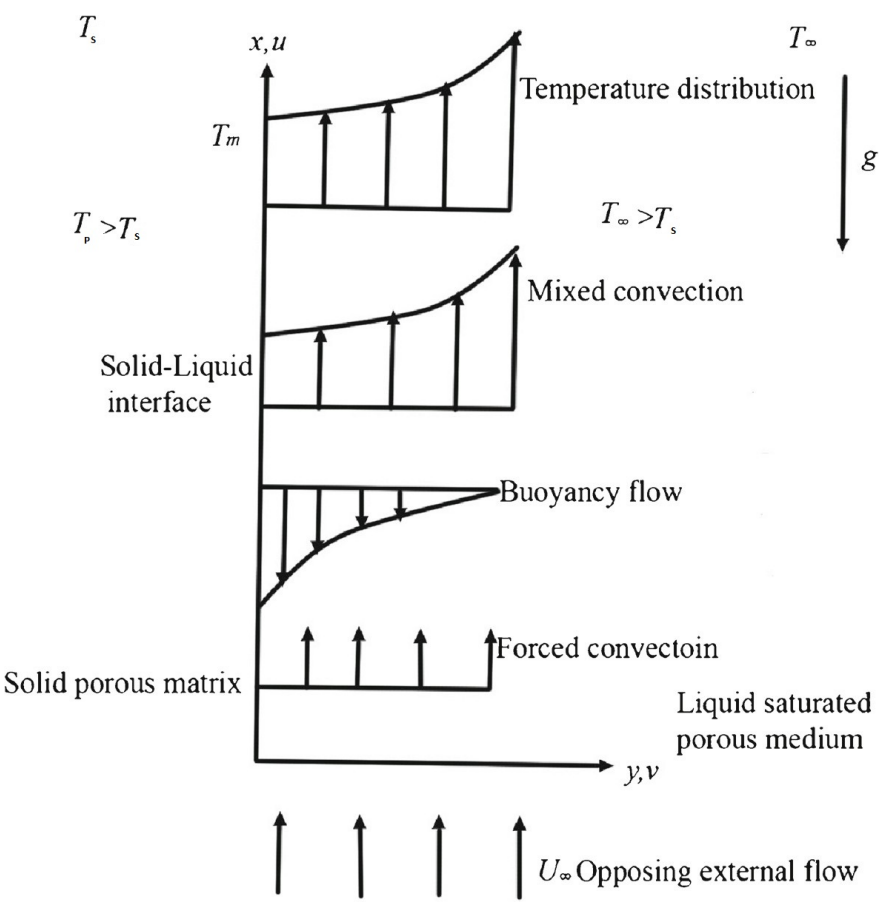

Fig. 1 Physical Model of the Problem (Hemalatha et al., 2015)

$$
\begin{gathered}
\frac{\partial u}{\partial x}+\frac{\partial v}{\partial y}=0 \\
\frac{\partial u}{\partial y}+\frac{c_{f} \sqrt{k}}{\nu} \frac{\partial}{\partial y}\left(u^{2}\right)=\frac{\rho g k}{\nu}\left(\beta_{T} \frac{\partial T}{\partial y}+\beta_{C} \frac{\partial C}{\partial y}\right) \\
u \frac{\partial T}{\partial x}+v \frac{\partial T}{\partial y}=\frac{\partial}{\partial y}\left(\alpha_{\tau} \frac{\partial T}{\partial y}\right)-\frac{1}{\rho c_{p}} \frac{\partial Q_{r}}{\partial y}+\frac{\mu}{\rho c_{p}}\left(\frac{\partial u}{\partial y}\right)^{2} \\
u \frac{\partial C}{\partial x}+v \frac{\partial C}{\partial y}=\frac{\partial}{\partial y}\left(D_{s} \frac{\partial C}{\partial y}\right)-K_{n}\left(C-C_{\infty}\right)^{n}
\end{gathered}
$$

where $u, v$ are velocity components along $\mathrm{x}, \mathrm{y}$ directions respectively, $c_{f}$ is the Forchheimer constant, $\nu$ is kinematic viscosity of the fluid, $g$ is acceleration due to gravity, $k$ is permeability of the porous medium, $c_{p}$ is specific heat at constant pressure, $\beta_{T}$ is coefficient of thermal expansion, $\beta_{C}$ is coefficient of solute expansion, $D_{s}$ is thermal solute diffusivity, $K_{n}$ is chemical reaction rate of order $n, \rho$ is fluid density and $C_{\infty}$ is free stream concentration, $\mu$ is dynamic viscosity of the fluid. The boundary conditions which govern the fluid flow are

$$
\begin{gathered}
k_{e t} \frac{\partial T}{\partial y}=\rho\left[L+c_{s}\left(T_{p}-T_{s}\right)\right] v, T=T_{p}, C=C_{w}, \text { at } y=0 \text { and } \\
u \rightarrow u_{\infty}, T \rightarrow T_{\infty}, C \rightarrow C_{\infty} \text { as } y \rightarrow \infty
\end{gathered}
$$

where $L, c_{s}$ are latent heat of solid and specific heat of solid phase respectively. $C_{w}$ is concentration of fluid near the wall and $u_{\infty}$ is free stream velocity. In presence of mechanical dispersion one can write thermal diffusivity $\alpha_{\tau}=\alpha_{m}+\alpha_{d}$ where $\alpha_{m}$ is molecular diffusivity and $\alpha_{d}=$ $D u d$, is dispersion thermal diffusivity. Further solutal diffusivity $D_{s}=$ $D_{m}+D_{d}$ where $D_{m}$, is molecular solutal diffusivity and $D_{d}=\beta u d$, is dispersion solutal diffusivity. $D$ and $\beta$ are proportionality constants and $d$, is particle mean diameter. The effective thermal conductivity of the porous medium $k_{e t}=\alpha_{\tau} \rho c_{p}$. Assume further the temperature gradient is sufficiently small within the flow, allows us to represent $T^{4}$ as a linear function of temperature. Then the variation in radiative heat flux based on Rosseland's approximation may be modeled as

$$
\frac{\partial Q_{r}}{\partial y}=-\frac{16 \widetilde{\sigma} T_{p}^{3}}{3 k^{*}} \frac{\partial T}{\partial y}
$$

where $\widetilde{\sigma}$, is the Stefan-Boltzmanconstant, $k^{*}$, is mean absorption coefficient. Define a stream function $\psi=f(\eta) \sqrt{\alpha_{m} u_{\infty} x}$ which satisfies $u=\frac{\partial \psi}{\partial y}$ and $v=-\frac{\partial \psi}{\partial x}$. Velocity components in non dimension form may be derived as

$$
u=u_{\infty} f^{\prime}(\eta), \quad v=-\frac{1}{2} \sqrt{\frac{\alpha_{m} u_{\infty}}{x}}\left[f(\eta)-\eta f^{\prime}(\eta)\right]
$$

where $\eta$ is the similarity variable defined by

$$
\eta(x, y)=\frac{y}{x} \sqrt{\frac{u_{\infty} x}{\alpha_{m}}}
$$

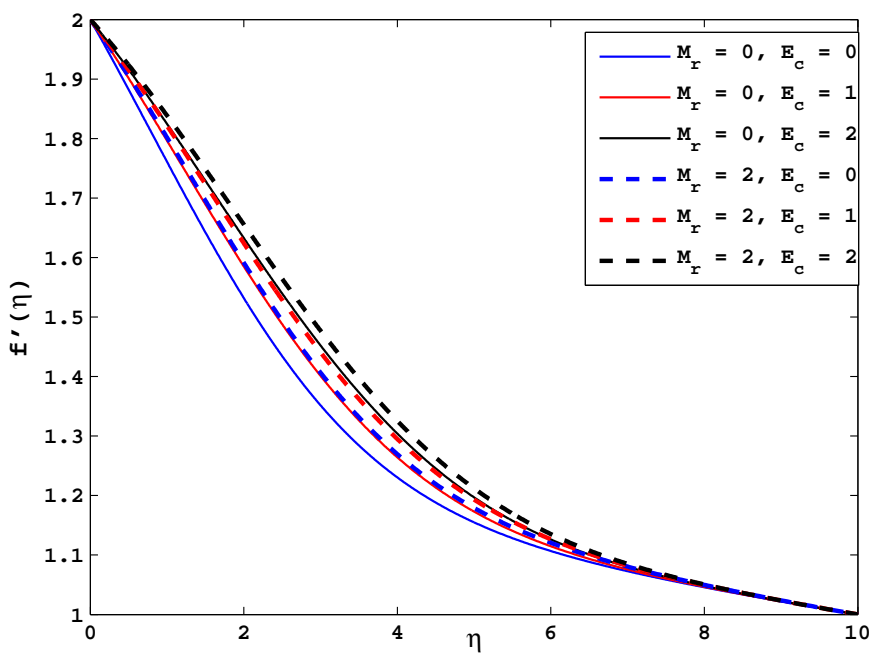

Fig. 2 Melting and viscous dissipation effects on velocity profile for $R a / P e=F_{r}=N_{r}=2, N_{b}=\gamma=\gamma^{*}=L e=n=\Gamma=1$

Further introduce another set of non-dimensional quantities, temperature and concentration, as

$$
\theta(\eta)=\frac{T-T_{p}}{T_{\infty}-T_{p}}, \quad \phi(\eta)=\frac{C-C_{w}}{C_{\infty}-C_{w}}
$$

The equations (6)-(9) transform equations (2)-(5) in the following undimensional form

$$
\begin{gathered}
f^{\prime \prime}\left(1+F_{r} f^{\prime}\right)=-\frac{R a}{P e}\left(\theta^{\prime}+N_{b} \phi^{\prime}\right) \\
\theta^{\prime \prime}\left(1+\gamma f^{\prime}+\frac{4}{3} N_{r}\right)=-\frac{1}{2} f \theta^{\prime}-\gamma f^{\prime \prime} \theta^{\prime}-\operatorname{PrEc} f^{\prime \prime 2} \\
\phi^{\prime \prime}\left(\frac{1}{L e}+\gamma^{*} f^{\prime}\right)=-\gamma^{*} \phi^{\prime} f^{\prime \prime}-\frac{1}{2} f \phi^{\prime}+\Gamma \phi^{n}
\end{gathered}
$$




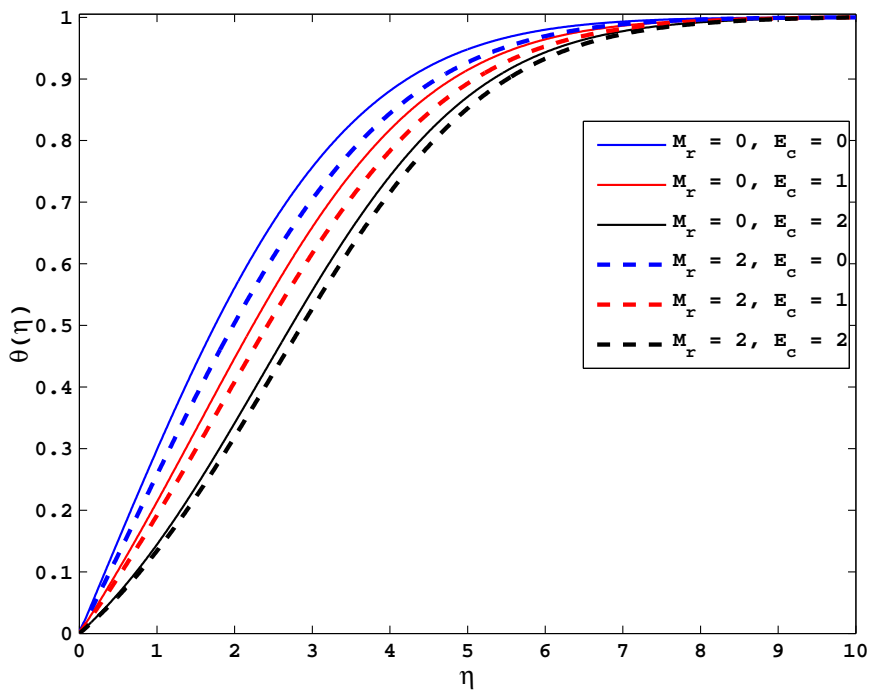

Fig. 3 Melting and viscous dissipation effects on temperature profile for $R a / P e=F_{r}=N_{r}=2, N_{b}=\gamma=\gamma^{*}=L e=n=\Gamma=1$

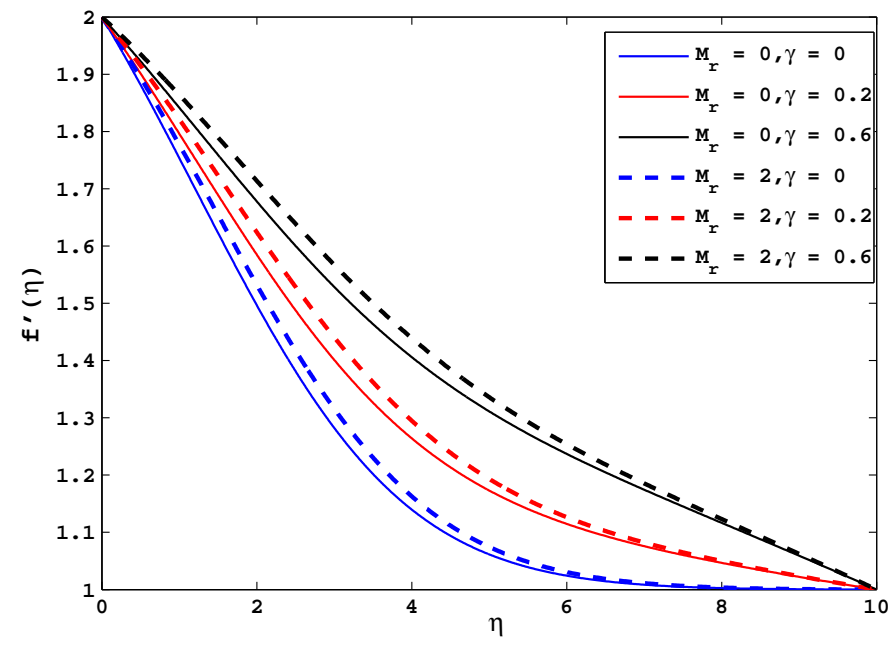

Fig. 4 Melting and thermal dispersion effects on velocity profile for $R a / P e=F_{r}=N_{r}=2, N_{b}=\gamma^{*}=L e=n=\Gamma=E c=1$

along with relevant boundary conditions

$$
f(0)+2 M_{r} \theta^{\prime}(0)=0, \theta(0)=0, \phi(0)=0
$$

and

$$
f^{\prime}(\infty)=1, \theta(\infty)=1, \phi(\infty)=1
$$

where $R a=\frac{k g \beta_{T} \rho\left(T_{\infty}-T_{p}\right)}{\nu \alpha_{m}}, P e=\frac{u_{\infty}}{\alpha_{m}}$; Eckert number $E c=\frac{u_{\infty}^{2}}{c_{p} \Delta T}$; non-Darcy parameter $F_{r}=\frac{2 c_{f} \sqrt{k} u_{\infty}}{\nu}$; thermal dispersion parameter $\gamma=$ $\frac{D d u_{\infty}}{\alpha_{m}}$; buoyancy parameter $N_{b}=\frac{C_{w}-C_{\infty}}{T_{p}-T_{\infty}}$; radiation parameter $N_{r}=$ $\frac{4 \widetilde{\sigma} T_{p}^{3}}{k_{e t} k^{*}}$; Prandtl number $\operatorname{Pr}=\frac{\nu}{\alpha_{m}}$; Lewis number $L e=\frac{\alpha_{m}}{D_{m}}$; solute dispersion parameter $\gamma^{*}=\frac{\beta d u_{\infty}}{\alpha_{m}}$; modified chemical reaction parameter $\Gamma=\frac{K_{n} x\left(C_{\infty}-C_{w}\right)^{n-1}}{u_{\infty}}$ and melting parameter $M_{r}=\frac{c_{f}\left(T_{\infty}-T_{p}\right)}{L+c_{s}\left(T_{p}-T_{0}\right)}$.

Nusselt and Sherwood numbers witness the nature of heat and mass transfer respectively in a fluid flow and are defined by

$$
\begin{aligned}
N u_{x} & =\frac{x q_{w}(x)}{k_{e t}\left(T_{p}-T_{\infty}\right)} \\
S h_{x} & =\frac{x \widehat{m}(x)}{D_{s}\left(C_{\infty}-C_{w}\right)}
\end{aligned}
$$

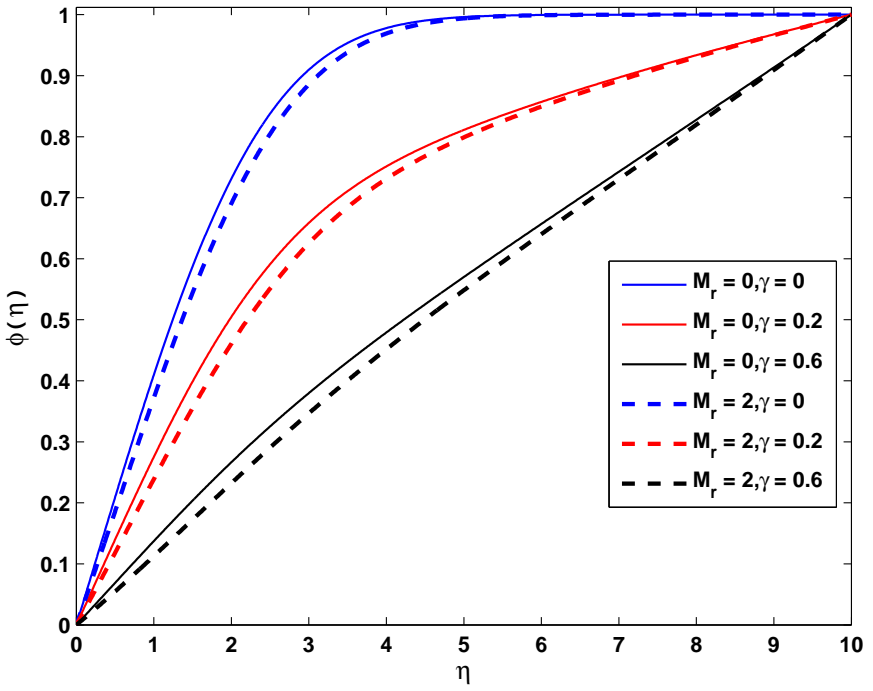

Fig. 5 Melting and thermal dispersion effects on velocity profile for $R a / P e=F_{r}=N_{r}=2, N_{b}=E c=\gamma^{*}=L e=n=\Gamma=1$

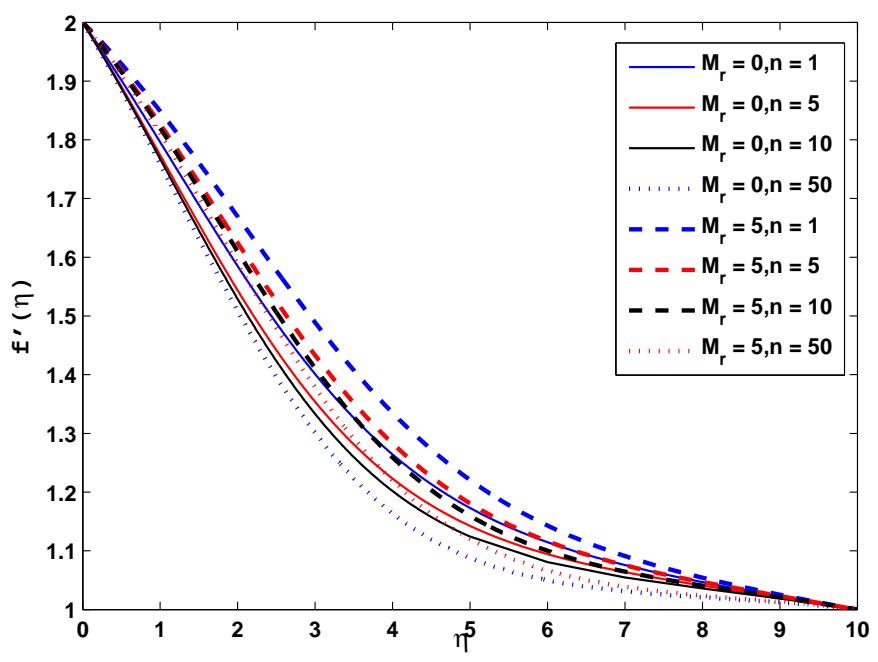

Fig. 6 Melting and order of chemical reaction effects on velocity profile for $R a / P e=F_{r}=N_{r}=2, N_{b}=\gamma=\gamma^{*}=L e=E c=$ $\Gamma=1$

where

$$
\begin{aligned}
q_{w}(x) & =-\left.k_{e t} \frac{\partial T}{\partial y}\right|_{y=0} \\
\widehat{m}(x) & =-\left.D_{s} \frac{\partial C}{\partial y}\right|_{y=0}
\end{aligned}
$$

provide heat and mass flux at the wall respectively. Equations (8), (9), (14)-(17) yield the following dimensionless local Nusselt and Sherwood numbers respectively.

$$
\begin{gathered}
\frac{N u_{x}}{\sqrt{P e_{x}}}=-\left[1+\frac{4}{3} N_{r}+\gamma f^{\prime}(0)\right] \theta^{\prime}(0) \\
\frac{S h_{x}}{\sqrt{P e_{x}}}=-\left[1+\gamma^{*} f^{\prime}(0)\right] \phi^{\prime}(0)
\end{gathered}
$$

\section{NUMERICAL SOLUTION WITH MATLAB-BVP4C SOLVER}

\subsection{Introduction to bvp4c}

BVP4C enforces a collocation method for the solution of a boundary value problem (BVP) of the form $y^{\prime}=f(x, y, c)$ subject to general non- 


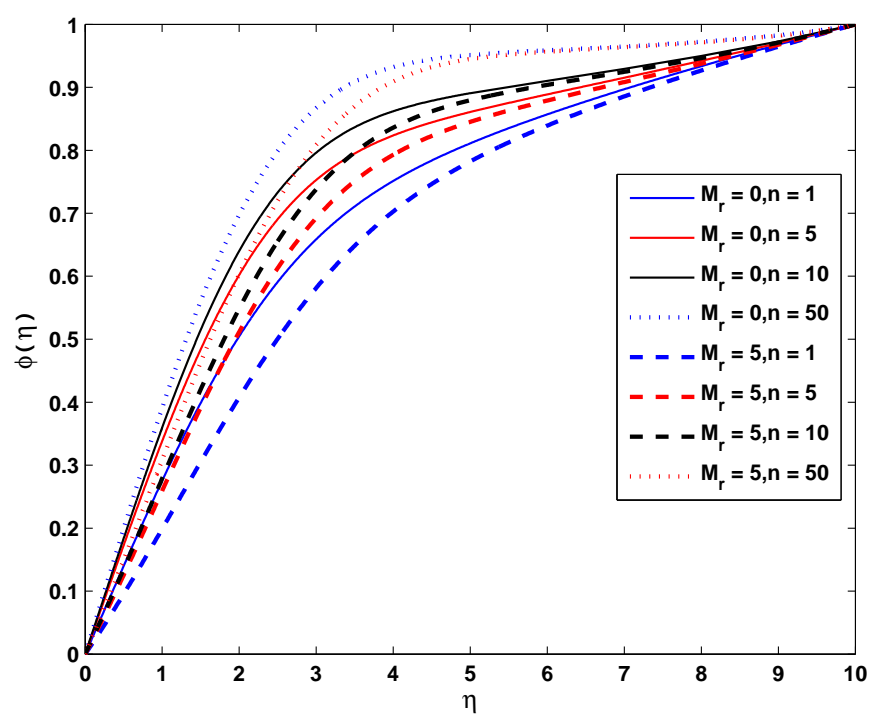

Fig. 7 Melting and order of chemical reaction effects on concentration profile for $R a / P e=F_{r}=N_{r}=2, N_{b}=\gamma=\gamma^{*}=L e=$ $E c=\Gamma=1$

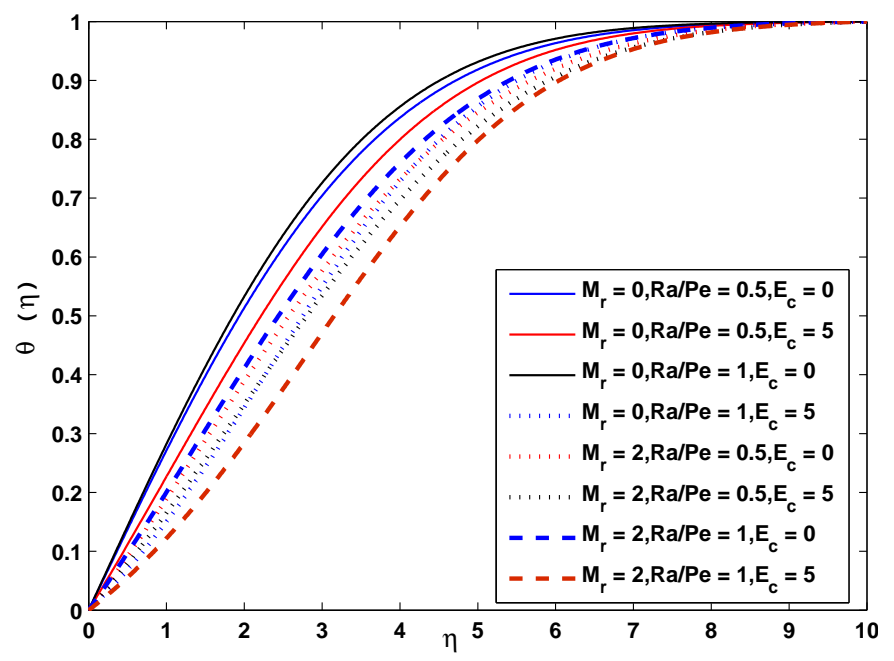

Fig. 8 Mixed convection along with Melting effects on temperature for $F_{r}=N_{r}=2, N_{b}=\gamma=\gamma^{*}=L e=n=\Gamma=1$

linear, two point boundary conditions $g(y(a), y(b), c)=0$ where $a \leq$ $x \leq b$. The methodology involves the following two key stages(Jacek and Lawrence, 2001).

Stage1. Approximate solution $\mathbf{S}(\mathbf{x})$

A cubic polynomial function $\mathrm{S}(\mathrm{x})$ is defined over each interval $\left[x_{n}, x_{n+1}\right]$ of each mesh $a=x_{0}<x_{1}<---<x_{n}=b$, satisfying the boundary conditions $g(S(a), S(b))=0$. Also $\mathrm{S}(\mathrm{x})$ satisfies the collocates at both ends and the mid points of each sub interval. $S^{\prime}\left(x_{n}\right)=f\left(x_{n}, S\left(x_{n}\right)\right)$ $S^{\prime}\left(\frac{x_{n}+x_{n+1}}{2}\right)=f\left[\frac{x_{n}+x_{n+1}}{2}, S\left(\frac{x_{n}+x_{n+1}}{2}\right)\right]$ and

$S^{\prime}\left(x_{n+1}\right)=f\left(x_{n+1}, S\left(x_{n+1}\right)\right)$. These are solved iteratively by Simpson's method. With modest assumptions, $\mathrm{S}(\mathrm{x})$ may be a fourth order approximation to an isolated solution $\mathrm{y}(\mathrm{x}),\|\mid y(x)-S(x)\| \| \leq C h^{4}$ where $h$ is the maximum of step sizes $h_{n}=x_{n+1}-x_{n}$ and $C$ is constant. Once $\mathrm{S}(\mathrm{x})$ is evaluated on a mesh with bvp4c, it can be found inexpensively at any $\mathrm{x}$ in $[\mathrm{a}, \mathrm{b}]$ with 'bvpval' function.

Stage2. Residual function $\mathbf{r}(\mathbf{x})$

To control the error, define the residual in ordinary differential equation as $r(x)=S^{\prime}(x)-f[x, S(x)]$ with boundary condition $g(S(a), S(b))$.

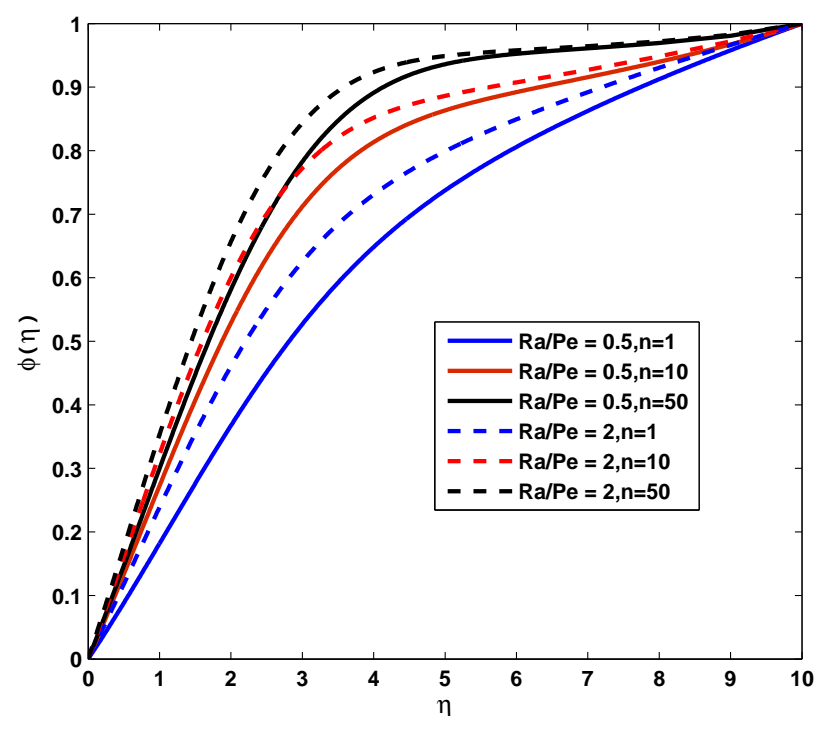

Fig. 9 Mixed convection and order of chemical reaction effects on concentration profile for $M_{r}=F_{r}=N_{r}=2, N_{b}=\gamma=\gamma^{*}=$ $L e=E c=\Gamma=1$

If the residuals are uniformly small, $\mathrm{S}(\mathrm{x})$ is a good solution.

\subsection{Numerical solution to boundary layer equations with bvp4c}

The set of coupled non-linear ordinary differential equations (10)-(12) are reduced to a set of first order differential equations as follows: $f_{1}^{\prime}=f_{2} ; f_{2}^{\prime}=-\left(\frac{R a}{P e}\right)\left(\frac{f_{4}+N_{b} f_{6}}{1+F_{r} f_{2}}\right)$;

$f_{3}^{\prime}=f_{4} ; f_{4}^{\prime}=-\left(\frac{1}{1+\gamma f_{2}+\left(\frac{4}{3}\right) N_{r}}\right)\left(\frac{1}{2} f_{1} f_{4}+\gamma f_{2}^{\prime} f_{4}+\operatorname{PrEc} f_{2}^{2}\right) ;$

$f_{5}^{\prime}=f_{6} ; f_{6}^{\prime}=-\left(\frac{L e\left[\gamma^{*} f_{6} f_{2}^{\prime}+\frac{1}{2} f_{1} f_{6}+\Gamma f_{5}^{n}\right]}{1+L e \gamma^{*} f_{2}}\right)$

where $f_{1}=f, f_{2}=f^{\prime}, f_{3}=\theta, f_{4}=\theta^{\prime}, f_{5}=\phi, f_{6}=\phi^{\prime}$ Further the boundary conditions (13) are noted as $f_{1}(0)+2 M_{r} f_{4}(0)=$ $0, f_{3}(0)=0, f_{5}(0)=0$ and $f_{2}(\infty)=1, f_{3}(\infty)=1, f_{5}(\infty)=1$ The absolute and relative errors of tolerance on the residuals are set by options $=$ bvpset $\left({ }^{\prime}\right.$ AbsTol' $, 1 e-10,{ }^{\prime}$ RelTol, $\left.1 e-10\right)$

Initial value problems are solved by 'bvpinit' solinit $=$ bvpinit $\left(\right.$ linspace $\left.(0,10,10),\left[\begin{array}{ll}0 & 10\end{array}\right]\right)$.

The following code executes the boundary value problem along with boundary conditions $s o l=b v p 4 c($ file_ode, file_bc, solinit,options $)$ $d y d x=\left[\quad y_{2}\right.$

$$
-(R a / P e) *\left(y_{4}+N_{b} * y_{6}\right) /\left(1+F_{r} * y_{2}\right)
$$

$y_{4}$

$\left(-0.5 * y_{1} * y_{4}+\gamma * y_{4} *(R a / P e) *\left(y_{4}+N_{b} * y_{6}\right) /\left(1+F_{r} * y_{2}\right)-\operatorname{Pr} *\right.$ $\left.E c *\left((R a / P e) *\left(y_{4}+N_{b} * y_{6}\right) /\left(1+F_{r} * y_{2}\right)\right)^{2}\right) /\left(1+\gamma * y_{2}+(4 / 3) * N_{r}\right)$ $y_{6}$

$\left(-0.5 * y_{1} * y_{6}+\gamma^{*} * y_{6} *(R a / p e) *\left(y_{4}+N_{b} * y_{6}\right) /\left(1+F_{r} *\right.\right.$ $\left.\left.\left.y_{2}\right)+\Gamma * y_{5}^{n}\right) /\left(\gamma^{*} * y_{2}+(1 / L e)\right)\right]$

Residual is calculated by the following syntax: res $=$ file_bc(ya,yb) Advantages:

1. bvp4c is not a shooting method

2. The bound $h$ hold for all $x$ in $[a, b]$

3. Once $S(x)$ is found on a mesh with bvp4c, it can be computed inexpensively at any value of $\mathrm{x}$ in $[a, b]$

4. bvp4c is based an algorithms that are credible even when the initial mesh is very poor, yet furnish the correct results as $h \rightarrow 0$

5. The solution $\mathrm{y}(\mathrm{x})$ is approximated over the whole interval $[a, b]$ and the boundary conditions are taken into account at all times, which is not in the case of shooting method. 


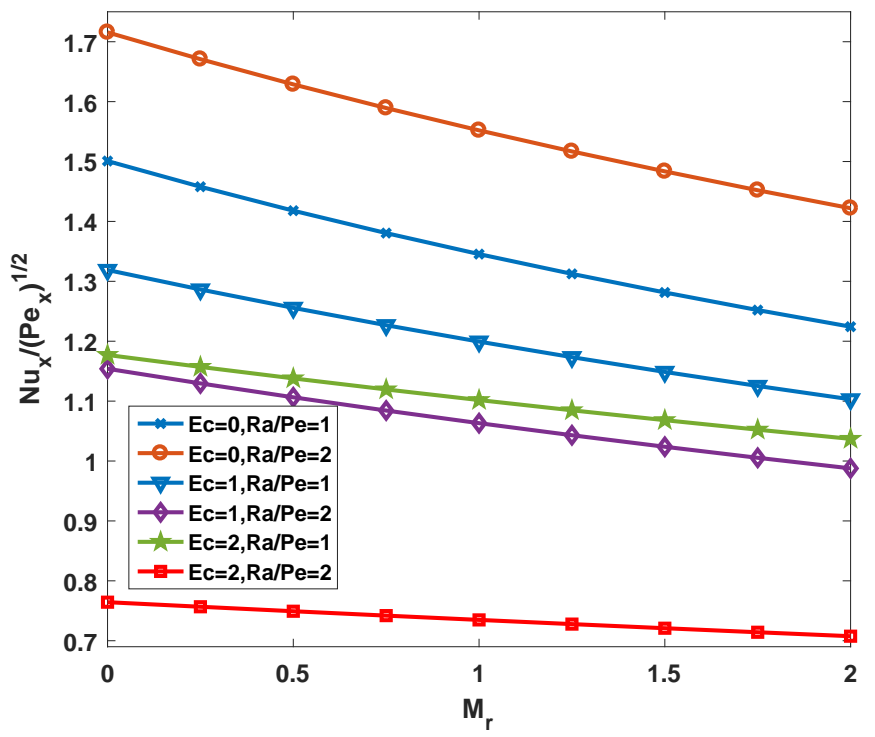

Fig. 10 Heat transfer against Melting parameter for $F_{r}=N_{r}=2, N_{b}=$ $\gamma=\gamma^{*}=L e=n=\Gamma=1$

\section{RESULTS AND DISCUSSION}

In the present study, the physics of the problem is mainly focused on the combined effect of viscous dissipation and melting parameter on fluid characteristics. Prandtl number is fixed as 6.2 for comparative study. Fig. 2 depicts the impact of melting parameter together with Eckert number on velocity profile. It is observed that increasing the value of melting parameter shows an increment in both momentum boundary layer thickness and velocity profile. Further,for a fixed $M_{r}$, Eckert number enhances the velocity profile.

The theoretical phenomena is as follows: Kinetic energy will be converted to internal energy by work done against viscous stream representing viscous dissipation. The heat developed during this process enhances the velocity of the fluid particles. So more viscous dissipation triggers more velocity. From Fig. 3, descents are found in both thermal boundary layer and temperature profiles on increasing melting parameter. This may be due to the convective heat transfer is inhibited from liquid-saturated porous medium to the solid plate. A similar profile is observed in the case of Eckert number too. Effect of thermal dispersion on momentum and thermal boundary layers are notified in Fig. 4 and Fig. 5. Boundary layer thickness decreases with increase in the value of thermal dispersion. Also fluid particles attain momentum by increasing the dispersion. Further increasing thermal dispersion shows a decrement in fluid concentration level.

The influence of order of chemical reaction on velocity and concentration profiles are witnessed in Fig. 6 and Fig. 7. Increasing chemical reaction parameter reduces the fluid velocity and enhances the concentration profiles. Since it is known that higher order reactions will produce a kind of resistance and hence momentum will be reduced. Fig. 8 shows the effect of temperature on mixed convection along with viscous dissipation and melting parameter. Temperature is enhanced with increasing mixed convection parameter. The decrement in temperature profiles on enhancing Eckert number are more dominant in forced convection $(R a / P e=1)$. In presence of melting these variations are getting lower.

Fig. 9 depicts the intensity of chemical reaction on concentration profiles at various convection parameters. It is noticed that increasing mixed convection parameter yields an increase in concentration profiles. Further an increase in order of reaction will cause more momentum in

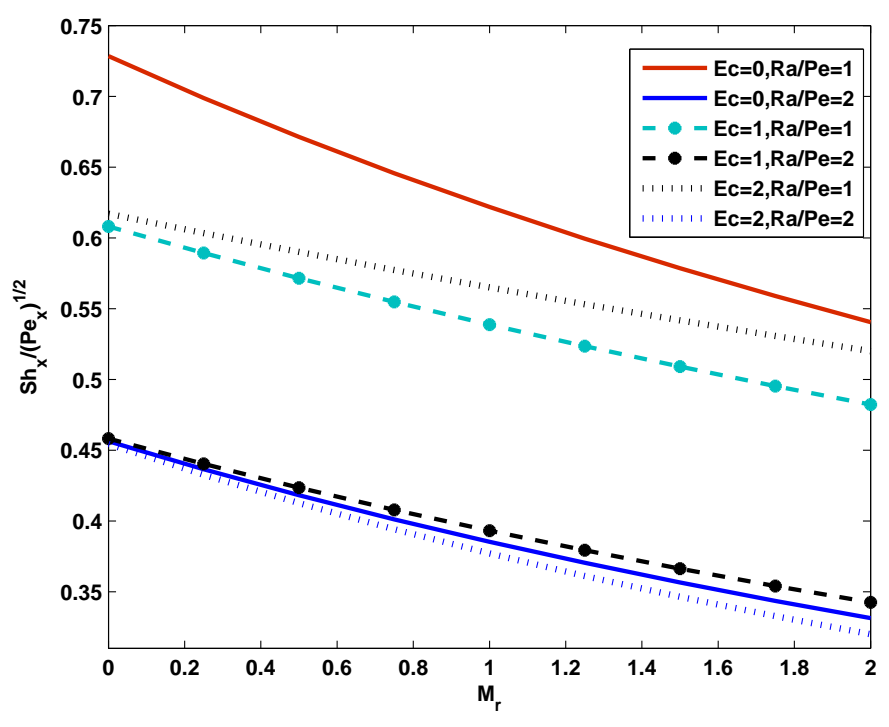

Fig. 11 Mass transfer against melting parameter for $F_{r}=N_{r}=2, N_{b}=$ $\gamma=\gamma^{*}=L e=n=\Gamma=1$

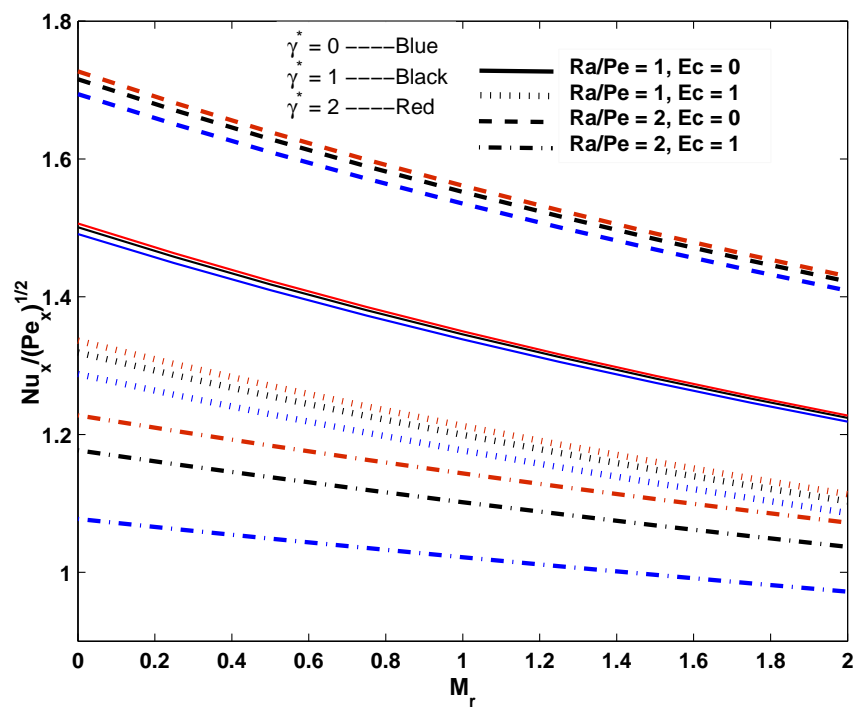

Fig. 12 Nusselt number against melting parameter for $F_{r}=N_{r}=$ $2, N_{b}=\gamma=L e=n=\Gamma=1$

the molecules so that species will diffuse more rapidly yielding increase in concentration levels. The same can be viewed from this figure.

Fig. 10 renders the effect of melting on Nusselt number. It is evident from this figure that a fall in heat transfer occurs with increase in melting parameter. For a fixed $M_{r}$, it is observed that viscous dissipation resists the rate of heat transfer whereas mixed convection assists. Fig. 11 shows the effect of Sherwood number, a quantitative measure of mass transfer, on melting. In absence of melting $\left(M_{r}=0\right)$, viscous dissipation has no effect on mass transfer in the case of mixed convection, but the same is notable for forced convection $(R a / P e=1$, upper stream).

Sherwood number decreases with increasing melting parameter. Also we found the mass transfer is high in inviscid flows $(E c=0)$. The effect of solute dispersion on heat and mass transfer is described in Fig. 12 and Fig. 13 respectively. Nusselt number increases with increasing solute dispersion. This is very significant in a mixed convective viscous fluid $(R a / P e=2, E c=1)$. Fig. 13 points out the solute dispersion enhances the local Sherwood number. It is observed from the same figure that this variation is more significant in presence of dispersion. Table 1 


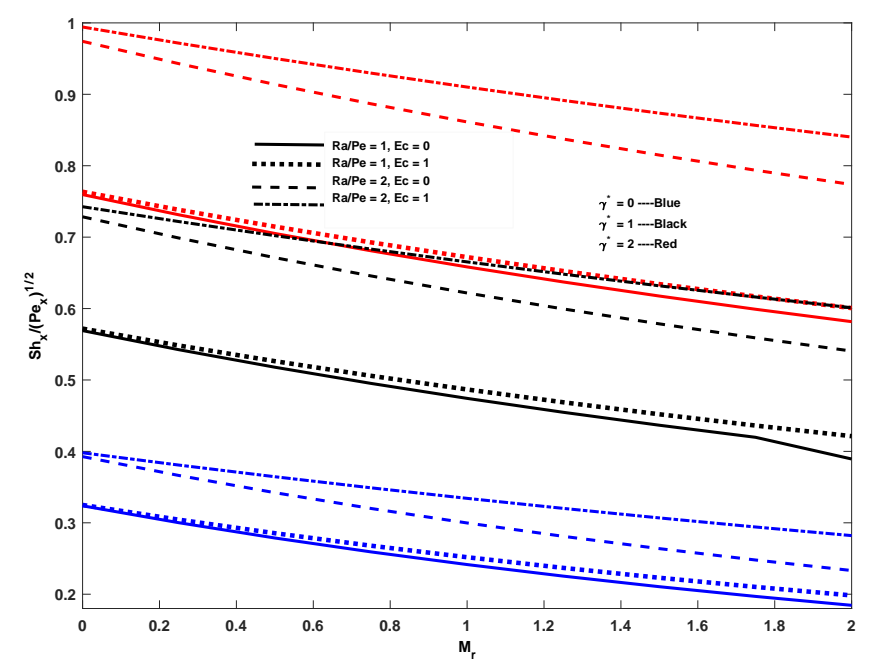

Fig. 13 Sherwood number against melting parameter for $F_{r}=N_{r}=$ $2, N_{b}=\gamma=L e=n=\Gamma=1$

Table 1 Comparision of $f^{\prime}(0)$ with previous work for $F_{r}=N_{r}=N_{b}=$ $\gamma=\gamma^{*}=L e=E c=0$

\begin{tabular}{|c|c|c|}
\hline $\mathrm{Ra} / \mathrm{Pe}$ & $\mathrm{f}^{\prime}(0)$ (Hemalatha et al., 2015) & $\mathrm{f}^{\prime}(0)$ (present) \\
\hline 0.0 & 1.000 & 1.000 \\
\hline 1.4 & 2.400 & 2.400 \\
\hline 3.0 & 4.000 & 4.000 \\
\hline 8.0 & 9.000 & 9.000 \\
\hline 10.0 & 11.00 & 11.00 \\
\hline 20.0 & 21.00 & 21.00 \\
\hline
\end{tabular}

and Table 2 show the agreement of the results with previous work in absence of viscous dissipation.

\section{CONCLUSIONS}

A two dimensional incompressible viscous fluid over a flat vertical plate is studied under chemical reaction and melting effect. The flow equations are numerically solved by means of MATLAB bvp4c solver. Results are obtained graphically. Melting causes an increase in velocity and decrease in temperature profiles. Viscous dissipation enhances velocity and reduces temperature profiles. Concentration decreases by increasing thermal dispersion. Solute dispersion increases both heat and mass transfer rates.

\section{ACKNOWLEDGEMENTS}

Author is very much thankful to the reviewers for their valuable comments and constructive suggestions to improve the quality of the paper.

\section{NOMENCLATURE}

$C \quad$ Concentration of the fluid

$c_{f} \quad$ Forchheimer constant

$c_{p} \quad$ specific heat

$c_{s} \quad$ Specific heat of solid phase

$c_{w} \quad$ Concentration of the wall

$D_{d} \quad$ Dispersion solutal diffusivity

$D_{m} \quad$ Molecular solutal diffusivity

$D_{s} \quad$ Thermal solute diffusivity

$d \quad$ Particle mean diameter

$g \quad$ Acceleration due to gravity

$K_{n} \quad$ Chemical reaction rate of order $n$
Table 2 Comparision of $\theta^{\prime}(0)$ with previous work for $F_{r}=N_{b}=\gamma=$ $\gamma^{*}=L e=E c=0$

\begin{tabular}{|c|c|c|}
\hline $\mathrm{Ra} / \mathrm{Pe}$ & $\theta^{\prime}(0)$ (Hemalatha et al., 2015) & $\theta^{\prime}(0)$ (present) \\
\hline 0.0 & 0.2706 & 0.2706 \\
\hline 1.4 & 0.3801 & 0.3801 \\
\hline 3.0 & 0.4745 & 0.4746 \\
\hline 8.0 & 0.6902 & 0.6901 \\
\hline 10.0 & 0.7594 & 0.7594 \\
\hline 20.0 & 1.0383 & 1.0383 \\
\hline
\end{tabular}

$k \quad$ Permeability of porous medium

$k_{e t} \quad$ Effective thermal conductivity

$k^{*} \quad$ Mean absorption coefficient

$L \quad$ Latent heat of solid

$Q_{r} \quad$ Radiative heat flux

$R a \quad$ Rayleigh number

$P e \quad$ Peclet number

$T \quad$ Temperature of the fluid

$T_{p} \quad$ Temperature of the plate

$T_{s} \quad$ Temperature of solid phase far from interface

$(u, v) \quad$ Velocity components along $x, y$ axes respectively

Greek Symbols

$\alpha_{T} \quad$ Thermal diffusivity

$\alpha_{m} \quad$ Molecular diffusivity

$\alpha_{d} \quad$ Dispersion thermal diffusivity

$\beta_{T} \quad$ Coefficient of thermal expansion

$\beta_{C} \quad$ Coefficient of solute expansion

$\eta \quad$ Similarity variable

$\rho \quad$ fluid density

$\mu \quad$ dynamic viscosity

$\nu \quad$ Kinematic viscosity

$\widetilde{\sigma} \quad$ Stefan Boltzman constant

Subscripts

$\infty \quad$ ambient enviroment

\section{REFERENCES}

Abbas, I.A., El-Amin, M.F., and Salman, A., 2008, “Combined Effect of Thermal Dispersion and Radiation on Free Convection in a Fluid Saturated, Optically Thick Porous Medium," Forsch Ingenieurwes, 72, 135144

https://doi.org/10.1007/s10010-008-0075-y

Ahmad, S., and Pop, I., 2014, "Melting Effect on Mixed Convection Boundary Layer Flow about a Vertical Surface Embedded in a Porous Medium opposing flows case," Transp. Por. Med., 102(3), 317-323. https://doi.org/10.1007/s11242-014-0291-x.

Aydin, O., and Kaya, A., 2008, "Radiation Effect on MHD Mixed Convection Flow about a Permeable Vertical Plate," Heat Mass Transfer, 45, 239-246.

https://doi.org/10.1007/s00231-008-0428-y

Chaudhary, R.C., Sharma, B.K., and Jha, A.K., 2006, "Radiation Effect with Simultaneous Thermal and Mass Diffusion in MHD Mixed Convection Flow," Rom. J. Phys., 51, 715-727.

Cheng, W.T., and Lin, C.H., 2007, "Melting Effect on Mixed Convective Heat Transfer with Aiding and Opposing External Flows from the Vertical Plate in a Liquid-Saturated Porous Medium," Int. J. Heat Mass Transf., 50, 3026-3034.

https://doi.org/10.1016/j.ijheatmasstransfer.2006.12.018 
Epstein, M., Cho, D.H., 1976, "Laminar Film Condensation on a Vertical Melting Surface," ASME J. Heat Transf., 98, 108-113.

https://doi.org/10.1115/1.3450451

Gebhart, B., Jaluria, Y., Mahajan, R.L., and Sammakia, B., 1989, "Buoyancy Induced Flows and Transport," J. Electornic Packaging, 111(4), 321. https://doi.org/10.1115/1.3226555

Gorla, R.S.R., Mansour, M.A., Hassanien, I.A., and Bakier, A.Y., 1999, "Mixed Convection Effect on Melting from a Vertical Plate in a Porous Medium," Transp. Por. Med.,36(2), 245-254. https://doi.org/10.1023/A:1006566924390

Hemalatha, K., Kameswaran, P.K., and Madhavi, M.V.D.N.S., 2015, "Mixed Convective Heat Transfer from a Vertical Plate Embedded in a Saturated Non-Darcy Porous Medium with Concentration and Melting Effect," Sadhana, 40, 455-465.

https://doi.org/10.1007/s12046-015-0373-7

Hossain, M.A., and Takhar, H.S., 1996, "Radiation Effect on Mixed Convection along a Vertical Plate with Uniform Surface Temperature," Heat Mass Transf.,31, 243-248.

https://doi.org/10.1007/BF02328616

Kandasamy, R., Abd Wahid Raj, B.Md., Khamis Azme, B., 2006, "Effects of Chemical Reaction, Heat and Mass Transfer on Boundary Layer Flow over a Porous Wedge with Heat Radiation in the Presence of Suction or Injection," Theoretical and Applied Mechanics , 33(2), 123-148. https://doi.org/10.2298/TAM0602123K

Jacek, K., and Lawrence, F. Shampine., 2001, “A BVP Solver Based on Residual Control and the MATLAB PSE," ACM Transactions on Mathematical Software, 27(3), 299-316.

https://doi.org/10.1145/502800.502801
Noor, N.F.M., Abbasbandy, S., and Hashim, I., 2012, "Heat and Mass Transfer of Thermophoretic MHD Flow over an Inclined Radiate Isothermal Permeable Surface in the Presence of Heat Source/Sink," Int. J. Heat Mass Transf, 55, 2122-2128.

https://doi.org/10.1016/j.ijheatmasstransfer.2011.12.015

Prasad, B.D.C.N., and Hemalatha, K., 2010, "Non-Darcy Mixed Convection with Thermal Dispersion-Radiation in a Saturated Porous Medium," Open Transp. Phenom. J., 2, 109-115.

Roberts, L., 1958, "On the Melting of a Semi-Infinite Body of Ice Placed in a Hot Stream of Air," J. Fluid Mech., 4, 505-528. https://doi.org/10.1017/S002211205800063X

Seddeek, M.A., 2002, "Effects of Radiation and Variable Viscosity on a MHD Free Convection Past a Semi-Infnite Flat Plate with an Aligned Magnetic Field in the Case of Unsteady Flow," Int. J. Heat Mass Transfer, 45, 931-935.

https://doi.org/10.1016/S0017-9310(01)00189-2

Sobha, V.V., Vasudeva, R.Y., Ramakrishna, K., and Hemalatha, K., 2010, "Non-Darcy Mixed Convection with Thermal Dispersion in a Saturated Porous Medium," ASME J. Heat Transfer, 132(1), 1-4. https://doi.org/10.1115/1.3194761

Soundalgekar, V.M., Gupta, S.K., Birajdar, N.S., 1979, "Effects of Mass Transfer and Free Convection Currents on MHD Stokes Problem for a Vertical Plate," Nucl. Eng. Res., 53(3), 339-346. https://doi.org/10.1016/0029-5493(79)90060-8

Tien, C., and Yen, Y.C., 1965, "The Effect of Melting on Forced Convection Heat Transfer," J. Appl. Meteorology , 4, 523-527.

https://doi.org/10.1175/1520-0450(1965)004<0523:teomof>2.0.co;2 